\title{
Decomposition of Multi-Objective Evolutionary Algorithm based on Estimation of Distribution
}

\author{
Jian-Qiu Zhang*, Feng Xu and Xian-Wen Fang \\ School of Science, Anhui University of Science and Technology, Huainan 232001, P. R. China
}

Received: 11 Jun. 2013, Revised: 16 Oct. 2013, Accepted: 17 Oct. 2013

Published online: 1 Jan. 2014

\begin{abstract}
Decomposition of multi-objective evolutionary algorithm has better distribution, but the number of groups will increase dramatically as the target number increases, seriously affecting the efficiency of the algorithm. This paper presents a decomposition of multi-objective evolutionary algorithm based on estimation of distribution, the basic idea of which is: to decompose multiple objectives into several single objective first and then to establish the probability model for every single objective based on the idea of estimation of distribution, generating the solution by sampling. Numerical analysis and experiments show that the solution of the new algorithm not only has better diversity and uniformity, but also the computational complexity of the algorithm is significantly lower than the decomposition of multi-objective evolutionary algorithm, especially for optimization of three goals.
\end{abstract}

Keywords: Multi-objective optimization, evolutionary algorithm, decomposition strategy, estimation of distribution.

\section{Introduction}

Since 1985, Schaffer made the first multi-objective evolutionary algorithms Vector Evaluated Genetic Algorithms(VEGA) [1], scholars of all Nations have given different evolutionary multi-objective optimization algorithm one after another, among which Multi-objective Genetic Algorithm (MOGA) [2] by Fonseca and Fleming, Non-Dominated Sorting Genetic Algorithm (NSGA, NSGAII) [3] by Srinivas and Deb, Strength Pareto Evolutionary Algorithm (SPEA, SPEA2) [4] by Zitzler and Thiele, Niched Pareto Genetic Algorithm (NPGA, NPGA2) [5] by Horn and Nafpliotis are typical.

From 2003 up to now, new characteristics of research on evolutionary multi-objective algorithm could have been seen. some new dominant mechanisms and evolutionary mechanisms have emerged, which is distinct from traditional Pareto-dominated mechanisms. Laumanns and Deb put forward Pareto-Adaptive $\varepsilon$ dominance [6], and Alfredo and Coello Coello Pareto-Adaptive $\varepsilon$ dominance [7], Zhang and Zhou Regularity Model Based Multi-Objective Estimation of Distribution Algorithm(RM-MEDA) [8], Zhang and Li Multi-Objective Evolutionary Algorithm Based on Decomposition (MOEA/D) [9] by combining evolutionary algorithms with traditional mathematical programming methods. At present, the new dominant mechanisms, new evolution mechanism, high-dimensional multi-objective optimization and multi-objective optimization test have been the focus of research on evolutionary multi-objective optimization algorithm.

This paper researched the principle of RM-MEDA \& MOEA/D, proposed Regularity Model Based Multi-Objective Estimation of Distribution Algorithm and Decomposition Algorithm, use performance analysis and numerical simulation to proved the suppose.

\section{Decomposition of multi-objective evolutionary algorithm and estimation of distribution algorithms}

The basic idea of solving multi-objective optimization by traditional optimization algorithms is to transform various sub-goals after a weighted combination into single-objective optimization. Multi-objective evolutionary algorithms is to regard all targets as a whole so as to find as many representative evenly distributed Pareto optimal solutions as possible by appropriate evolutionary approach.

\footnotetext{
*Corresponding author e-mail: fzjq@ @ 163.com
} 
Zhang and $\mathrm{Li}$ introduced the idea of traditional multi-objective optimization algorithm into the multi-objective evolution algorithm, proposing MOEA/D. Decomposition of multi-objective evolutionary algorithm is to decompose the multi-objective optimization into several simple-target optimization, and at the same time evolve them as a community, each generation of community of the evolution being composed by the current optimal solutions of each sub target. In MOEA/D, optimization of each sub target only needs individual information of its neighbor. The neighbor relation among sub targets is decided by the distance between each weight vector of objective function. The more close the distance of weight vector between two sub-goals is, the more approximate their solutions will inevitably be. Thus it can be seen that whether weight vector of each objective function will fill the entire space and whether the distribution is even is the key problem in MOEA/D.

Estimation of distribution algorithms is an emerging branch in the field of evolutionary computation, which is the combination of evolutionary algorithms and statistical learning. The algorithms are to build individual distribution probability models in the solution space by statistical learning, and then to evolve the model with the idea of evolution. In Estimation of distribution algorithm there is no crossover and mutation operations, which is replaced by estimating probability model of solution space and generating the new group by sampling the probability model. Estimation of distribution algorithms grasps the direction of group evolution from a macro which can solve high-dimensional multi-objective optimization so as to reduce the time complexity effectively at the initial stages. In multi-objective optimization, it is impossible to optimize multiple goals at the same time, so the goal of optimization is to find a set of Pareto optimal solutions, which can be solved appropriately since estimation of distribution algorithm is suitable for solving such problems because of its inherent parallelism.

But basic difference between multi-objective optimization and the simple-target optimization lies in taking the influence of multiple goals into consideration in the meanwhile during the process of seeking optimization, thus causing the entire community to evolve in the direction that does not evolution which does not increase the function value of multiple goals. When solving simple-target optimization by estimation of distribution algorithms, the renewal of probability vector is carried on according to the distribution of the main body of the highest part of adaptation value, then the adaptation value of multiple goals should be considered simultaneously in the renewal of probability vector in multi-objective optimization. Therefore the order may be listed separately according to the adaptation value of each objective function to select multiple sub-communities as the best representatives of different goal compatibility separately, just as model worker delegation in various trades, and then renew the probability vector according to various subgroups.

With the development of estimation of distribution algorithm and the advantages revealed when this algorithm is used to solve certain problems, some multi-objective optimization algorithms based on the idea of estimation of distribution have been proposed one after another. Khan combined selective strategies in NSGA-II and Bayesian optimization algorithm (BOA) together, proposed multiple-objective Bayesian optimization algorithm (MBOA) [10], and achieved better results than NSGA-II. Laumanns combines SPEA2 and BOA for solving multi-objective Knapsack problem [11]. Scholars such as Zhang and Zhou introduce RM-MEDA [8], a more classic algorithm for solving multi-objective optimization by estimation of distribution algorithm.

Algorithm flow chart of estimation of distribution algorithms and decomposition of multi-objective evolutionary algorithm are shown in Fig.1 and Fig.2.

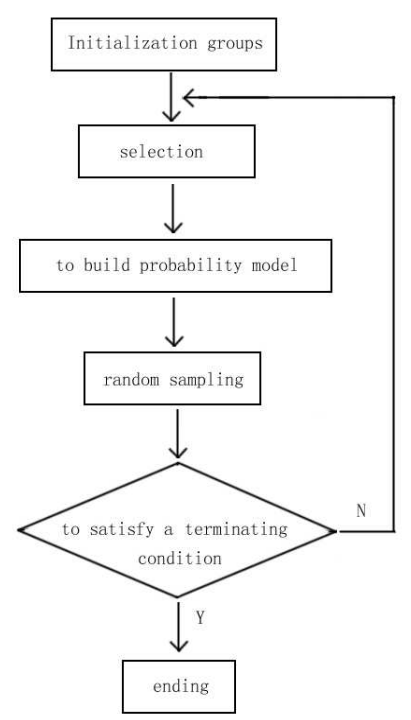

Fig. 1: Algorithm flow chart of estimation of distribution algorithms

\section{Decomposition of multi-objective evolutionary algorithm based on estimation of distribution}

In MOEA/D, community size $\mathrm{N}$ and the weight vector $\lambda^{1}, \ldots, \lambda^{N}$ are controlled by parameter $\mathrm{H}$, namely every weight value of each objective function in each weight vector is the got from $\{0 / H, 1 / H, \ldots H / H\}$, but the 


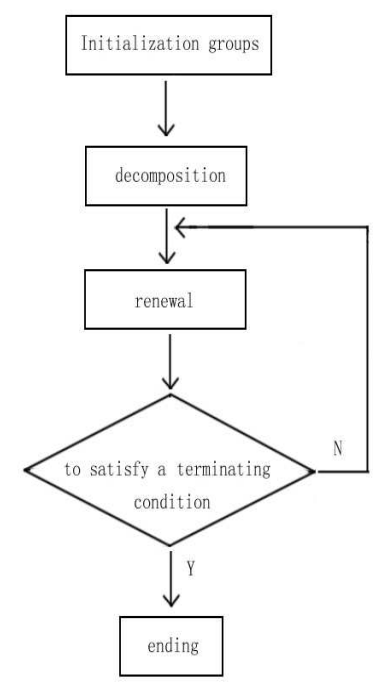

Fig. 2: decomposition of multi-objective evolutionary algorithm

number of weight vector $N=C_{H+m-1}^{m-1}$. When target figure $\mathrm{m}$ increases, $\mathrm{N}$ will increase rapidly; this will affect the operating efficiency of MOEA/D.

Considering that estimation of distribution algorithms can grasp the macro-group evolutionary direction, that there is no crossover and mutation operations, and that it can reduce time complexity very effectively during the initial phase, the paper presents DE-MOEA/D based on estimation of distribution, which uses probability models to evolve groups on the basis of the framework of MOEA/D.

There are a variety of methods that can transfer multi-objective optimization into a series of sub-optimization close to PF, such as boundary intersection method, Tchebycheff decomposition method, weights summation method. This algorithm is to use Tchebycheff decomposition method [12].

In each generation of populations, the quantity which the DE-MOEA/D algorithm needs to preserve includes:

(1) The population which has $N$ individuals $x^{1}, \ldots, x^{N} \in$ $\Omega$, in which $x_{i}$ is the current solution of the ith question;

(2) $F V^{1}, \ldots F V^{N}$, in which $F V^{i}$ is the function value of $x_{i}$, namely $F V^{i}=F\left(x_{i}\right), i=1, \ldots, N$;

(3) $z=\left(z_{1}, \ldots, z_{m}\right)^{T}$, in which $z_{i}$ is the optimal solution to the objective function $f_{i}$ which is found currently;

(4) Exterior population (EP) is used to preserve the non-control solution found in search process.

Processes of DE - MOEA/D are as follows:

Step1: Initialization:

Step1.1 to set $E P=\varphi$;

Step1.2 to calculate the Euclid distance between each two weight vectors and select the recent election $\mathrm{T}$ as its neighbors for each weight vector. That is, for each $i=1, \ldots, N$, there is $B(i)=\left\{i_{1}, \ldots i_{T}\right\}$ in which $\lambda^{i_{1}}, \ldots \lambda^{i_{T}}$ is the nearest $\mathrm{T}$ weight vector to $\lambda^{i}$;

Step1.3 to generate randomly the initial population $x^{1}, \ldots, x^{N} \in \Omega$ and set $F V^{i}=F\left(x^{i}\right)$;

Step 1.4 to set the initial value $z=\left(z_{1}, \ldots, z_{m}\right)^{T}$ according to the specific questions.

Step2: Renewal:

Step2.2 to calculate the different objective function values which are in correspondence with subgroups;

Step2.3 to establish a reasonable probability model according to the sub-group controlled by $\mathrm{T}$ closest weight vectors, and get new sample y based on this probability model;

Step2.4 renew z; for each $j=1, \ldots, m$, if $z_{j}<f_{j}\left(y^{\prime}\right)$, $z_{j}=f_{j}\left(y^{\prime}\right)$;

Step2.5 renew the groups; if $g\left(y^{\prime} \mid \lambda, z\right) \leq g\left(x^{j} \mid \lambda, z\right)$, for all $j \in B(i), x^{j}=y^{\prime}, F\left(v^{j}\right)=F\left(y^{\prime}\right)$;

Step2.6 renew EP; move all the solution controlled by $F\left(y^{\prime}\right)$ out of EP: if $F\left(y^{\prime}\right)$ is not dominated by any solution in EP, then move $F\left(y^{\prime}\right)$ into EP.

Step3: stop judgment; if the stopping criterion is satisfied it stops and output EP, otherwise return to Step2.

\section{Numerical experiments}

The following is numerical calculation on two standard test functions DTLZ1 and DTLZ2 by DE-MOEA/D and comparative analysis with NSGA-II to test the performance of DE-MOEA/D.

(1) DTLZ1 test function

$$
\begin{gathered}
\min _{1}(X)=\frac{1}{2} x_{1} x_{2} \ldots x_{M-1}\left[1+g\left(X_{M}\right)\right] \\
\operatorname{minf}_{2}(X)=\frac{1}{2} x_{1} x_{2} \ldots\left(1-x_{M-1}\right)\left[1+g\left(X_{M}\right)\right] \\
\vdots \\
\operatorname{minf}_{M-1}(X)=\frac{1}{2} x_{1}\left(1-x_{2}\right)\left[1+g\left(X_{M}\right)\right] \\
\operatorname{minf}_{M}(X)=\frac{1}{2}\left(1-x_{1}\right)\left[1+g\left(X_{M}\right)\right] \\
0 \leq x_{i} \leq 1, i=1,2, \ldots, n
\end{gathered}
$$

In which $0.5)])]$.

$$
g\left(x_{M}\right)=100\left[\left|x_{M}\right|+\sum_{x_{i} \in X_{M}}\left(\left(x_{i}-0.5\right)^{2}-\cos \left[2 0 \pi \left(x_{i}-\right.\right.\right.\right.
$$

When the test function achieves Pareto optimal boundary, all the corresponding $x_{i}$ belonging to $x_{m}$ is 0.5 , objective function value is on a linear hyper plane which satisfy $\sum_{m=1}^{M} f_{m}^{*}=0.5$. It is difficult for this problem to converge to Pareto optimal boundary because the corresponding search space includes $11^{k}-1 P F_{\text {local }}$, which will converge to the local optimal boundary generally. $P F_{\text {true }}$ When function value $M=3$ is shown in Fig.3. 


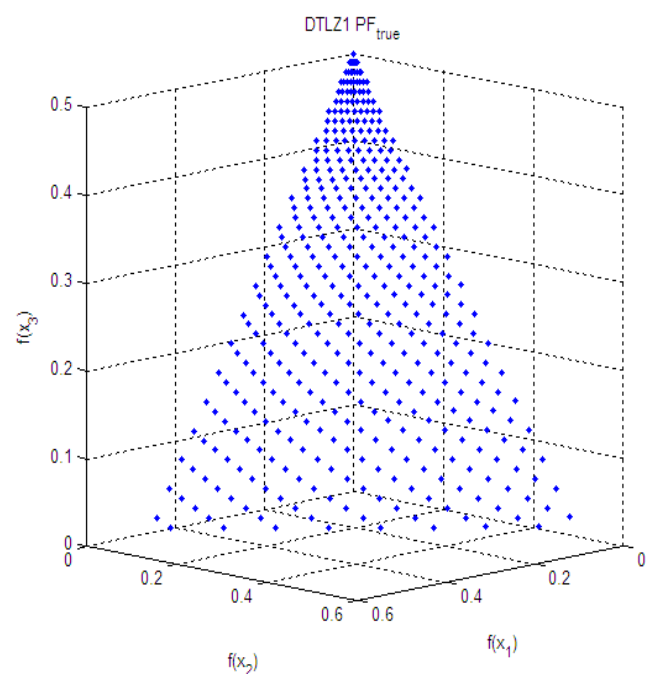

Fig. 3: DTLZ1 of PFtrue

(2) Test function in DTLZ2

$\left.\min _{1}(X)=\left[1+g\left(X_{M}\right)\right]\right) \cos \left(x_{1} \pi / 2\right) \ldots \cos \left(x_{M-2} \pi / 2\right) \cos \left(x_{M-1} \pi / 2\right)$ $\left.\min _{2}(X)=\left[1+g\left(X_{M}\right)\right]\right) \cos \left(x_{1} \pi / 2\right) \ldots \cos \left(x_{M-2} \pi / 2\right) \sin \left(x_{M-1} \pi / 2\right)$ $\left.\operatorname{minf}_{3}(X)=\left[1+g\left(X_{M}\right)\right]\right) \cos \left(x_{1} \pi / 2\right) \ldots \sin \left(x_{M-2} \pi / 2\right)$

$\left.\min _{M}(X)=\left[1+g\left(X_{M}\right)\right]\right) \sin \left(x_{1} \pi / 2\right)$ $0 \leq x_{i} \leq 1, i=1,2, \ldots, n$

In which $g\left(x_{M}\right)=\sum_{x_{i} \in X_{M}}\left(x_{i}-0.5\right)^{2}$

All $x_{i}$, belonging to $x_{M}$, to which Pareto optimal boundary of the test function corresponds is 0.5 , and the objective function value satisfies $\sum_{m=1}^{M} f_{m}^{2}=1$. This problem can be used to test operating capability of one MOEA. $P F_{\text {true }}$ when function value $M=3$ is a unit sphere within the first octant, as is shown in Fig.4.

It can be seen clearly from Figs.6 to 8 that DE-MOEA/D is obviously superior to NSGA-II in both distribution and uniform of Pareto optimal solutions, which shows that DE-MOEA/D inherit fully the advantages of distribution and uniformity of MOEA/D.

In order to test the convergence properties of DE-MOEA/D, the average value of IGD data of the last generation when DE-MOEA/D and MOEA/D are operated for 20 times is given in Table 1 .

Table 1: DE-MOEA/D and MOEA/Ds average IGD data

\begin{tabular}{c|ccc|ccc}
\hline \multirow{2}{*}{ Algorithm } & \multicolumn{3}{|c|}{ DTLZ1 } & \multicolumn{3}{c}{ DTLZ2 } \\
\cline { 2 - 7 } & Average & Minimum & $\begin{array}{c}\text { Standard } \\
\text { deviation }\end{array}$ & Average & & \\
\hline DE-MOEAmum & $\begin{array}{c}\text { Standard } \\
\text { deviation }\end{array}$ \\
\hline MOEA/D & 0.0254 & 0.0201 & 0.0018 & 0.0265 & 0.0259 & 0.0008 \\
\hline
\end{tabular}

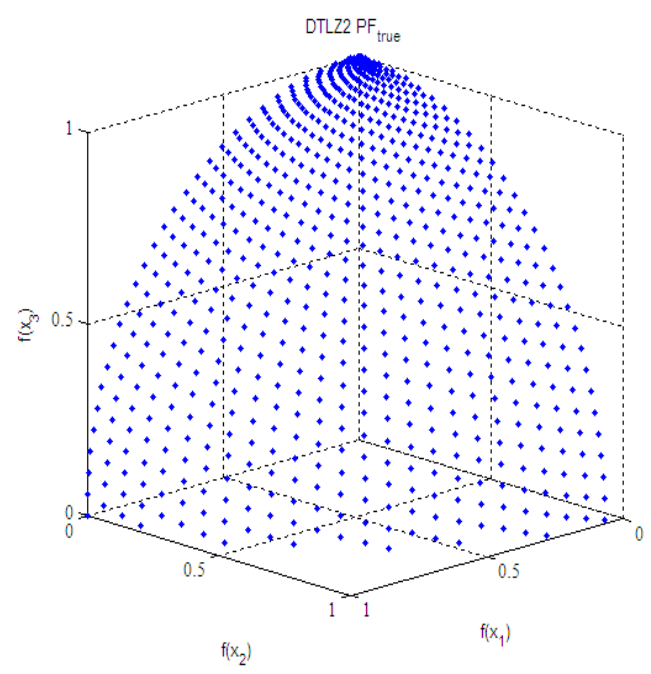

Fig. 4: DTLZ2 of PFtrue

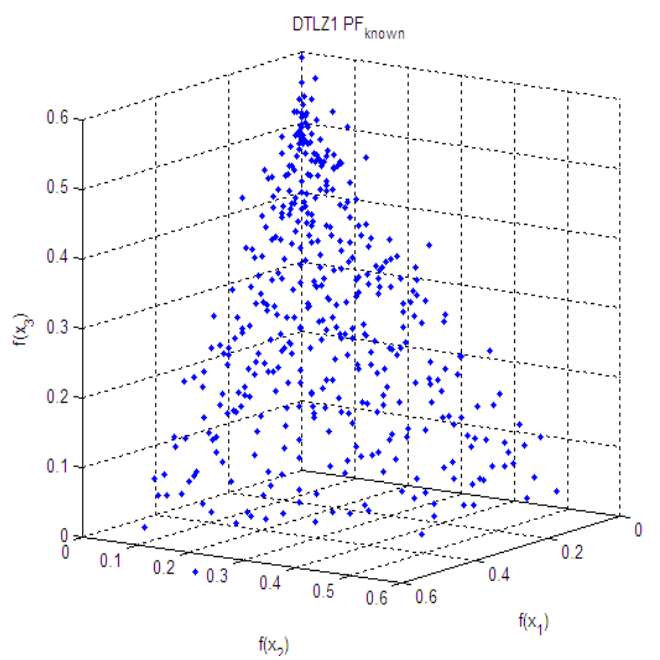

Fig. 5: DTLZ1s Pareto Optimal boundary with NSGA-II

The data in Table 1 clearly shows that convergence of DE-MOEA/D is faster than that of MOEA/D, that is, DE-MOEA/D is superior to MOEA/D in performance of calculation complexity.

\section{Conclusion}

This paper improves MOEA/D in view of the defect that calculation complexity increases rapidly when the number of objectives is rather large, proposes a new decomposition of multi-objective evolutionary algorithm based on estimation of distribution, and makes a 


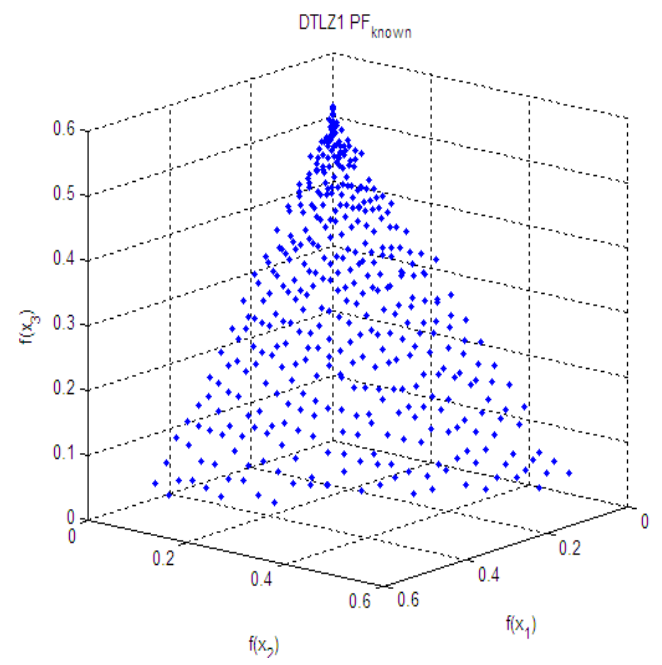

Fig. 6: DTLZ1s Pareto Optimal boundary with DE-MOEA/D

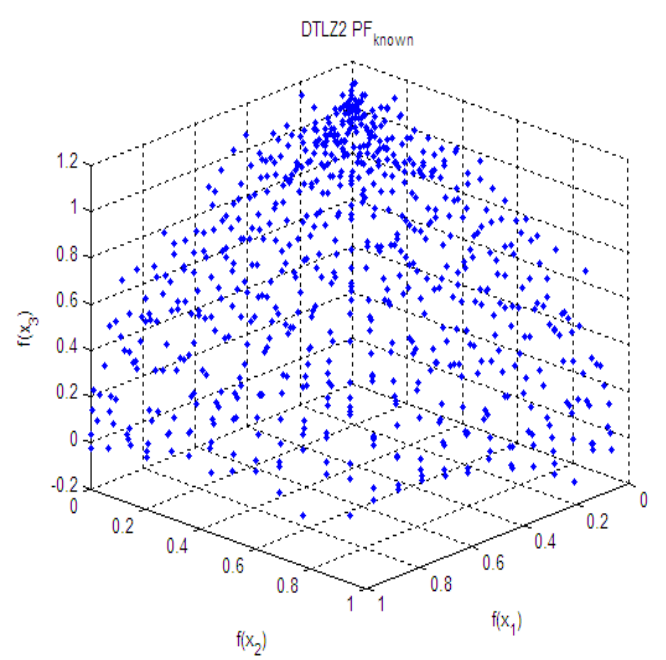

Fig. 7: DTLZ2s Pareto Optimal boundary with NSGA-II

comparative analysis with the current most outstanding NSGA-II.

Numerical analysis and experimental results show that the new algorithm is equivalent with MOEA/D but significantly superior to NSGA-II in distribution and uniformity of Pareto solution; As for the three-objective optimization, calculation complexity of the new algorithm is lower than that of MOEA/D because there is no traditional crossover and mutation operation in the new algorithm but a probability model which is used to generate evolutionary solution.

But some problems come up when the new algorithm is used in optimization of four objectives and the effect of optimization is less ideal. How to further improve the

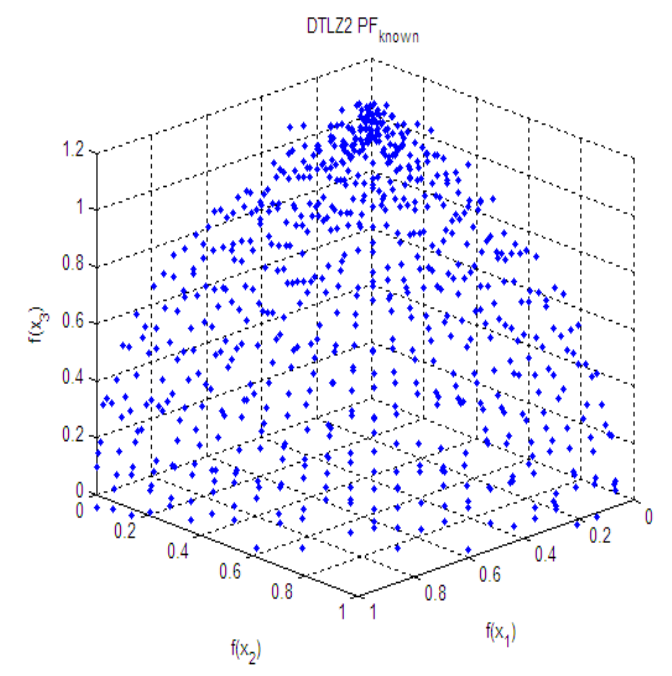

Fig. 8: DTLZ2s Pareto Optimal boundary with DE-MOEA/D

performance of decomposition of multi-objective evolutionary algorithm based on estimation of distribution to enable it to solve the higher dimensional multi-objective optimization will be our further research.

\section{Acknowledgement}

This work is partially supported by the National Natural Science Foundation of China (Grants 61272153 and 51274011), and the Excellent Young Staff Foundation of High School from Anhui Province (Grant 2012SQRL046). Thanks for the help.

\section{References}

[1] Schaffer JD. Multiple objective optimization with vector evaluated genetic algorithms. In: Grefenstette JJ, ed. Proc. of the Intl Conf. on Genetic Algorithms and Their Applications. Hillsdale: L. Erlbaum Associates, Inc., 93-100 (1985).

[2] Fonseca CM, Fleming PJ. Genetic algorithm for multiobjective optimization: Formulation, discussion and generation. In: Forrest S, ed. Proc. of the 5th Intl Conf. on Genetic Algorithms. San Mateo: Morgan Kauffman Publishers, 416-423 (1993).

[3] Deb K, Pratap A, Agarwal S, Meyarivan T. A fast and elitist multi-objective genetic algorithm: NSGA-II. IEEE Trans. on Evolutionary Computation, 6, 182-197 (2002).

[4] Zitzler E, Laumanns M, Thiele L. SPEA2: Improving the strength Pareto evolutionary algorithm. In: Giannakoglou K, Tsahalis DT, Priaux J, Papailiou KD, Fogarty T, eds. Evolutionary Methods for Design, Optimization and Control with Applications to Industrial Problems. Berlin: SpringerVerlag, 95-100 (2002). 
[5] Erickson M, Mayer A, Horn J. The niched Pareto genetic algorithm 2 applied to the design of groundwater remediation system. In: Zitzler E, Deb K, Thiele L, Coello Coello CA, Corne D, eds. Proc. of the 1st IntlConf. on Evolutionary Multi-Criterion Optimization, EMO 2001. Berlin: SpringerVerlag, 681-695 (2001).

[6] Laumanns M, Thiele L, Deb K, Zitzler E. Combining convergence and diversity in evolutionary multi-objective optimization. Evolutionary Computation, 10, 263-282 (2002).

[7] Hernndez-Dłaz AG, Santana-Quintero LV, Coello Coello CA, Molina J. Pareto-Adaptive -dominance. Evolutionary Computation, 15, 493-517 (2007).

[8] Zhang QF, Zhou AM, Jin Y. RM-MEDA: A regularity model based multiobjective estimation of distribution algorithm. IEEE Trans. on Evolutionary Computation, 12, 41-63 (2007).

[9] Zhang QF, Li H. MOEA/D: A multiobjective evolutionary algorithm based on decomposition. IEEE Trans. on Evolutionary Computation, 11, 712-731 (2007).

[10] Khan N, Goldberg DE, Pelican M. Multi-Objective Bayesian optimization algorithm. Technical Report, No.2002009, University of Illinois at Urbana-Champaign, (2002).

[11] Laumanns M, Ocenasek J. Bayesian optimization algorithms for multi-objective Optimization. In: Merelo JJ, Adamidis P, Beyer HG, eds. Proc. of the 7th Intl Conf. on Parallel Problem Solving from Nature. London: Springer-Verlag, 298-307, (2002).

[12] Miettinen K. Nonlinear Multiobjective Optimization. Norwell, MA: Kluwer, (1999).

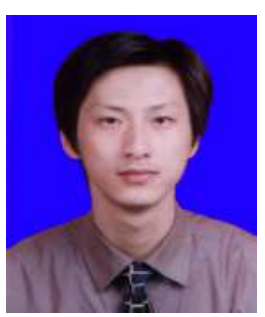

Jian-Qiu Zhang received the M.S degree in Electrical and Information Engineering from Anhui University of Science and Technology. $\mathrm{He}$ is currently a lecturer in Anhui University of Science and Technology. His research interests are in the areas of optimality theory and

Quantum information.

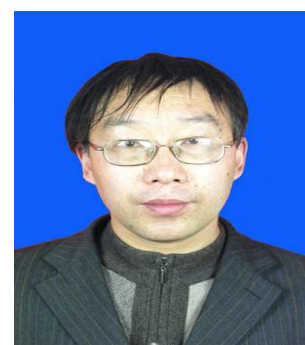

Feng $\mathbf{X u}$ received the M.S. degree in East China Normal University Education Ministry Key Laboratory of spectroscopy and spectroscopy. $\mathrm{He}$ is currently a Professor in Anhui University of Science and Technology. His research interests are in the areas of optimality theory and Nuclear Magnetic Resonance Spectroscopy.

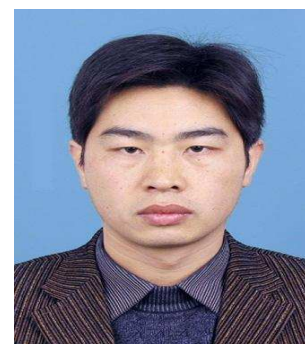

Xian-Wen

Fang received his M.A. degree from Shandong University of Science and Technology, China, in 2004, and $\mathrm{PhD}$. degree in the key Lab of Service Computing at Tongji University in 2011. He is currently a Professor with the Department of Computer Science and Engineering, Anhui University of Science and Technology, China. His research interests include Petri net, trustworthy software and Web services. $\mathrm{He}$ has published more than 60 papers in domestic and international academic journals and conference proceedings. These papers are embodied more than 40 times by SCI and EI and are cited more than 100 times by others. 